\title{
Ex post Nash consistent representation of effectivity functions
}

Citation for published version (APA):

Peters, H. J. M., Schröder, M. J. W., \& Vermeulen, A. J. (2013). Ex post Nash consistent representation of effectivity functions. Maastricht University, Graduate School of Business and Economics. GSBE Research Memoranda No. 049 https://doi.org/10.26481/umagsb.2013049

Document status and date:

Published: 01/01/2013

DOI:

10.26481/umagsb.2013049

Document Version:

Publisher's PDF, also known as Version of record

\section{Please check the document version of this publication:}

- A submitted manuscript is the version of the article upon submission and before peer-review. There can be important differences between the submitted version and the official published version of record.

People interested in the research are advised to contact the author for the final version of the publication, or visit the DOI to the publisher's website.

- The final author version and the galley proof are versions of the publication after peer review.

- The final published version features the final layout of the paper including the volume, issue and page numbers.

Link to publication

\footnotetext{
General rights rights.

- You may freely distribute the URL identifying the publication in the public portal. please follow below link for the End User Agreement:

www.umlib.nl/taverne-license

Take down policy

If you believe that this document breaches copyright please contact us at:

repository@maastrichtuniversity.nl

providing details and we will investigate your claim.
}

Copyright and moral rights for the publications made accessible in the public portal are retained by the authors and/or other copyright owners and it is a condition of accessing publications that users recognise and abide by the legal requirements associated with these

- Users may download and print one copy of any publication from the public portal for the purpose of private study or research.

- You may not further distribute the material or use it for any profit-making activity or commercial gain

If the publication is distributed under the terms of Article $25 \mathrm{fa}$ of the Dutch Copyright Act, indicated by the "Taverne" license above, 


\section{Maastricht University}

Hans Peters, Marc Schröder, Dries Vermeulen

\section{Ex post Nash consistent representations of effectivity functions}

RM/ 13/049

\section{GSBE}

Maastricht University School of Business and Economics

Graduate School of Business and Economics

P.O Box 616

NL-6200 MD Maastricht

The Netherlands 


\title{
Ex post Nash consistent representation of effectivity functions
}

\author{
Hans Peters*, Marc Schröder*, Dries Vermeulen*
}

This version, June 2013

\begin{abstract}
We consider effectivity functions for finitely many players and alternatives. We assume that players have incomplete information with respect to the preferences of the other players. Our main result is the characterization of effectivity functions which have an ex post Nash consistent representation, i.e., there is a game form such that (i) the distribution of power among coalitions of players is the same as in the effectivity function and (ii) there is an ex post Nash equilibrium (in pure strategies) for any preference profile.
\end{abstract}

Journal of Economic Literature Classification Nos. C62, C70, D82

Keywords Effectivity function, game form, incomplete information, ex post Nash consistent representation

\section{Introduction}

An effectivity function (Moulin and Peleg, 1982) describes the allocation of power among coalitions of individuals. More precisely, given a set of individuals and a set of alternatives, an effectivity function assigns to each coalition of individuals a collection of subsets of alternatives. Effectivity functions derive from many concepts in game theory and social choice theory: for instance game forms, social choice correspondences, simple games. More generally, effectivity functions can be used to describe the distribution of power or rights. For instance, a constitution can be modelled as a game

${ }^{*}$ Department of Quantitative Economics, Maastricht University, P.O. Box 616, 6200 MD Maastricht, The Netherlands. Email: m.schroeder@maastrichtuniversity.nl, h.peters@maastrichtuniversity.nl, d.vermeulen@maastrichtuniversity.nl 
form (Gärdenfors, 1981; Peleg, 1998): alternatives are social states, and to say that a coalition is effective for a set of alternatives means that this group of individuals is legally entitled to the social state being in the designated set.

According to this last interpretation an effectivity function is a centralized concept describing the rights of individuals. This leads directly to the question if we can find a set of decentralized rules (practical laws) such that individuals and coalitions, by acting according to these rules, obtain the same rights as described by the effectivity function? In more formal terms, can we find a game form which provides all coalitions with the same power as the effectivity function. This question was already answered in Moulin (1983), who showed that any monotonic and superadditive effectivity function can be represented by a game form. An important further question is if we can find such a representing game form that is stable in some sense. Peleg et al. (2002) answered this question by characterizing effectivity functions that have a Nash consistent representation, i.e., a game form representing the effectivity function and having a Nash equilibrium for any profile of preferences of the individuals. See Peleg and Peters (2010) for an extensive treatment of this topic and related issues.

An important open question in this area is the following. If we take into account that information may be incomplete - individuals are not sure about the preferences, i.e., types, of other individuals - when is it possible to have a representation of an effectivity function that is Bayesian Nash consistent? d'Aspremont and Peleg (1988) consider ordinal Bayesian incentive compatible representations of committees: they study effectivity functions derived from simple games and their representation by so-called decision schemes, which assign probability distributions over the alternatives, such that there exists a Bayesian incentive compatible Nash equilibrium under incomplete information. Recently, Peleg and Zamir (2013) show that, without additional restrictions except for the standard ones, effectivity functions can be Bayesian Nash consistently represented by such decision schemes; their proof of this result uses the uniform core (Abdou and Keiding, 1991). The question under which conditions such a representation by a deterministic game form exists, remains open.

In the present paper we impose a considerably stronger requirement: given an effectivity function, when does there exist a representing game form such that, for any information structure (i.e., vector of type sets of the players), there exists an ex post Nash equilibrium for any preference profile, that is, a strategy combination which results in a Nash equilibrium whatever the realization of the types? One the one hand, this is a very 
desirable situation, since players do not have to rely on their beliefs about the types of the others. On the other hand, as can be expected, this requirement turns out to be rather restrictive. Our main result is as follows. An effectivity function (for $n$ individuals or players) has an ex post Nash consistent representation exactly if there exists an $(n-1)$-player coalition and a subset $C$ of alternatives for which this coalition is effective, such that all other $(n-1)$-player coalitions are effective for all singletons in this set $C$. In the case $n=2$ this condition is equivalent to at least one of the two players being a so-called singleton player: a singleton player is a player for whom all minimal sets for which this player is effective, are singletons. We also show that, if only one of the players has more than one type, then ex post Nash consistency imposes no additional restrictions compared to Nash consistency (as in Peleg et al., 2002). In the final part of the paper we show that, even if an effectivity function does not have an ex post Nash consistent representation, it may still be the case that it has such a representation if the numbers of types are restricted - a special case being the mentioned one where only one player has more than one type. Throughout the paper we concentrate on existence of ex post Nash equilibria and leave other possible properties (e.g., Pareto optimality of such equilibria) from consideration.

The organization of the paper is as follows. Section 2 introduces definitions and notations. In Section 3, we present our basic representing game form. This game form is extended and modified in several ways in the rest of the paper. Section 4 analyzes the case of two players, and Section 5 generalizes to more than two players.

\section{The model}

Notation. For a finite set $D,|D|$ denotes the number of elements of $D$; $P(D)$ denotes the set of all subsets of $D ; P_{0}(D)$ denotes the set of all nonempty subsets of $D$.

Let $N=\{1, \ldots, n\}$ (where $n \geq 2$ ) be the set of players, and let $A$ be a finite set of alternatives, $|A| \geq 2$. A binary relation $R$ over $A$ is a subset $R \subseteq A \times A$, where $(a, b) \in R$ is written as $a R b$. A binary relation is complete if for all $a, b \in A$, we have $a R b$ or $b R a$. A binary relation is transitive if $a R b$ and $b R c$ jointly imply $a R c$, for all $a, b, c \in A$. A preference ordering $R$ on $A$ is a complete and transitive binary relation. The set of all preference orderings on $A$ is denoted by $W$. If $R \in W$ and $a, b \in A$, then $a P b$ means $a R b$ and not $b R a$. If $a \in A$ and $R \in W$, then $L(a, R)=\{b \in A \mid a R b\}$ is the lower contour set of $a$, i.e. the set of alternatives not strictly preferred to $a$. 
For a set $S, W^{S}=\{f \mid f: S \rightarrow W\}$ is the set of mappings from $S$ to $W$.

An effectivity function (EF) is a function $E: P(N) \rightarrow P\left(P_{0}(A)\right)$ that satisfies the following conditions: (i) $E(N)=P_{0}(A)$; (ii) $E(\emptyset)=\emptyset$; and (iii) $A \in E(S)$ for all $S \in P_{0}(N)$. As a general interpretation, $B \in E(S)$ means that coalition $S$ can force the final alternative to be an element of $B$. The interpretation of the three conditions is fairly obvious.

An effectivity function $E$ is monotonic (with respect to players as well as alternatives) if

$$
\left[B \in E(S), B^{\prime} \in P_{0}(A), B \subseteq B^{\prime} \text {, and } S \subseteq S^{\prime}\right] \Rightarrow B^{\prime} \in E\left(S^{\prime}\right) .
$$

For $B \in P_{0}(A)$ and $S \in P_{0}(N), B$ is minimal for coalition $S$ if $B \in E(S)$ and there is no $B^{\prime} \in E(S)$ such that $B^{\prime} \varsubsetneqq B$. A monotonic effectivity function $E$ is completely determined by these minimal sets.

The effectivity function $E$ is superadditive if

$$
\left[B_{i} \in E\left(S_{i}\right), i=1,2 \text {, and } S_{1} \cap S_{2}=\emptyset\right] \Rightarrow B_{1} \cap B_{2} \in E\left(S_{1} \cup S_{2}\right) .
$$

Note that a superadditive effectivity function is also monotonic with respect to players: for $S \subseteq S^{\prime}$ and $B \in E(S)$, superadditivity implies that $B=$ $B \cap A \in E\left(S \cup\left(S^{\prime} \backslash S\right)\right)=E\left(S^{\prime}\right)$. Monotonicity and superadditivity are natural properties in view of the interpretation given above. Moreover, effectivity functions derived from game forms have these properties.

The polar of $E$ is the effectivity function $E^{*}$ defined by: $E^{*}(\emptyset)=\emptyset$, and for $S \in P_{0}(N)$

$$
E^{*}(S)=\left\{B \in P_{0}(A) \mid B \cap B^{\prime} \neq \emptyset \text { for all } B^{\prime} \in E(N \backslash S)\right\} .
$$

Thus, if $B \in E^{*}(S)$, then the complementary coalition $N \backslash S$ cannot prevent $S$ from obtaining an alternative from $B$; in particular, $A \backslash B \notin E(N \backslash S)$.

$E$ is maximal if $E$ is superadditive and $E=E^{*}$. Note that a maximal effectivity function is also monotonic. Indeed, a superadditive effectivity function is monotonic with respect to players. Also, if $B \in E^{*}(S)$ and $B \subseteq C$, then obviously $C \cap B^{\prime} \neq \emptyset$ for all $B^{\prime} \in E(N \backslash S)$ and thus $C \in E^{*}(S)$. Hence if $E$ is maximal then $E$ is monotonic with respect to alternatives since $E^{*}$ is.

The following results are well-known (e.g., Peleg and Peters, 2010; Boros et al., 2010; or Crama and Hammer, 2011). For completeness, we provide the proofs.

Proposition 2.1. Let $E$ be a monotonic effectivity function. The following statements are equivalent: 
(i) $E=E^{*}$.

(ii) For all $B \in P_{0}(A)$ and for all $S \in P_{0}(N), B \in E(S)$ if and only if $B \cap B^{\prime} \neq \emptyset$ for all $B^{\prime} \in E(N \backslash S)$.

(iii) For all $B \in P_{0}(A)$ and for all $S \in P_{0}(N), B \in E(S)$ if and only if $A \backslash B \notin E(N \backslash S)$.

Proof. The implication $(i) \Rightarrow(i i)$ is obvious. To prove the implication (ii) $\Rightarrow\left(\right.$ iii), assume $(i i)$. If $B \in E(S)$ for some $B \in P_{0}(A)$ and $S \in P_{0}(N)$, then, by $(i i), A \backslash B \notin E(N \backslash S)$. Conversely, if $A \backslash B \notin E(N \backslash S)$ for some $B \in P_{0}(A)$ and $S \in P_{0}(N)$, then by (ii) there must be some $B^{\prime} \in E(S)$ such that $(A \backslash B) \cap B^{\prime}=\emptyset$. Clearly, $B^{\prime} \subseteq B$, so that $B \in E(S)$, again by (ii).

Finally, to prove the implication (iii) $\Rightarrow(i)$, assume (iii). If $B \in E^{*}(S)$ for some $B \in P_{0}(A)$ and $S \in P_{0}(N)$, then $A \backslash B \notin E(N \backslash S)$, hence $B \in E(S)$ by (iii). Conversely, if $B \in E(S)$ for some $B \in P_{0}(A)$ and $S \in P_{0}(N)$, then $A \backslash B \notin E(N \backslash S)$ by (iii). Let $B^{\prime} \in E(N \backslash S)$, then $B^{\prime} \nsubseteq A \backslash B$ by monotonicity, so that $B \cap B^{\prime} \neq \emptyset$. So $B \in E^{*}(S)$.

Remark 2.2. Note that, in the proof of Proposition 2.1, monotonicity of $E$ is only used to prove that $E(S) \subseteq E^{*}(S)$ for all $S \in P(N)$ in the last part of the proof. This would also follow directly from superadditivity of $E$ : if $B \in E(S)$ then superadditivity would imply $B \cap B^{\prime} \neq \emptyset$ for all $B^{\prime} \in E(N \backslash S)$, so that $B \in E^{*}(S)$. Hence, Proposition 2.1 also holds with superadditivity instead of monotonicity.

The following result will be useful in the sequel.

Proposition 2.3. Let $E$ be a maximal effectivity function. Then for each minimal $B \in E(S)$ and alternative $a \in B$, there is a minimal $B^{\prime} \in E(N \backslash S)$ such that $B \cap B^{\prime}=\{a\}$.

Proof. Let $S \in P_{0}(N), B \in E(S)$, and $a \in B$. By superadditivity, $B \cap B^{\prime} \neq \emptyset$ for all $B^{\prime} \in E(N \backslash S)$. Suppose that for each minimal $B^{\prime} \in E(N \backslash S)$ there is some $b \neq a$ such that $b \in B \cap B^{\prime}$. Then $(B \backslash\{a\}) \cap B^{\prime} \neq \emptyset$ for all minimal $B^{\prime} \in E(N \backslash S)$, hence for all $B^{\prime} \in E(N \backslash S)$. Thus, $B \backslash\{a\} \in E^{*}(S)=E(S)$. So $B$ is not minimal in $E(S)$.

A game form is an $(n+2)$-tuple $\Gamma=\left(\Sigma^{1}, \ldots, \Sigma^{n} ; \pi ; A\right)$, where (i) $\Sigma^{i}$ is the (non-empty, finite) set of possible actions ${ }^{1}$ of player $i \in N$; and (ii)

\footnotetext{
${ }^{1}$ We reserve the term 'strategy' for game forms with incomplete information later on.
} 
$\pi: \Sigma^{1} \times \cdots \times \Sigma^{n} \rightarrow A$ is the outcome function. Throughout the paper we assume that $\pi$ is surjective. For $S \in P_{0}(N)$ we denote $\Sigma^{S}=\prod_{i \in S} \Sigma^{i}$.

Let $\Gamma=\left(\Sigma^{1}, \ldots, \Sigma^{n} ; \pi ; A\right)$ be a game form. For $S \in P_{0}(N)$ and $\sigma^{S} \in \Sigma^{S}$, we define $B\left(\sigma^{S}\right)=\left\{\pi\left(\sigma^{S}, \sigma^{N \backslash S}\right) \mid \sigma^{N \backslash S} \in \Sigma^{N \backslash S}\right\}$. The effectivity function $E^{\Gamma}$, associated with $\Gamma$, is defined in the following way: $E^{\Gamma}(\emptyset)=\emptyset$ and for $S \in P_{0}(N)$,

$$
E^{\Gamma}(S)=\left\{B \in P_{0}(A) \mid B\left(\sigma^{S}\right) \subseteq B \text { for some } \sigma^{S} \in \Sigma^{S}\right\} .
$$

Note that $E^{\Gamma}$ is monotonic and superadditive. Let $E: P(N) \rightarrow P\left(P_{0}(A)\right)$ be an effectivity function. A game form $\Gamma$ is a representation of $E$ if $E(S)=$ $E^{\Gamma}(S)$ for every $S \in P_{0}(N)$. Basically, this means that the game form distributes the same power among the players as the effectivity function does.

Remark 2.4. If $B \in P_{0}(A)$ is a minimal set of coalition $S \in P_{0}(N)$ in $E^{\Gamma}(S)$, then there exists an action profile $\sigma^{S} \in \Sigma^{S}$ such that $B\left(\sigma^{S}\right)=B$.

An information structure is an $n$-tuple $T=\left(T^{1}, \ldots, T^{n}\right)$, where $T^{i}$ is the finite set of types of player $i=1, \ldots, n$. We denote by $T^{N}=\prod_{i \in N} T^{i}$ the set of type profiles. With some abuse of notation we denote by $W^{T}$ the set of all preference ordering profiles: a profile $R^{T} \in W^{T}$ has dimension $\sum_{i \in N}\left|T^{i}\right|$ and assigns a preference ordering to each type of each player. If $\Gamma=\left(\Sigma^{1}, \ldots, \Sigma^{n} ; \pi ; A\right)$ is a game form, then $\left(\Gamma, T, R^{T}\right)$ is a game of incomplete information in the sense of Harsanyi (1967). The set of strategies of player $i$ in this game is the set $S^{i}=\left\{s^{i} \mid s^{i}: T^{i} \rightarrow \Sigma^{i}\right\}$. We denote $S^{N}=\prod_{i \in N} S^{i}$. We do not introduce type probabilities since we will only consider ex post Nash equilibrium. A strategy profile $s=\left(s^{1}, \ldots, s^{n}\right) \in S^{N}$ is an ex post (Nash) equilibrium (EPE) of $\left(\Gamma, T, R^{T}\right)$ if for all $i \in N$, all $t=\left(t^{1}, \ldots, t^{n}\right) \in T^{N}$ and all $\sigma^{i} \in \Sigma^{i}$,

$$
\pi(s(t)) R^{t^{i}} \pi\left(s^{-i}\left(t^{-i}\right), \sigma^{i}\right),
$$

where $s^{-i}\left(t^{-i}\right)$ is the vector $\left(s^{j}\left(t^{j}\right)\right)_{j \neq i}$. Let $s \in S^{N}$ be an EPE, then we call $\pi(s(t))$ the Nash outcome for $t \in T^{N}$. Game form $\Gamma$ is ex post (Nash) consistent for $T$ if $\left(\Gamma, T, R^{T}\right)$ has an EPE for every $R^{T} \in W^{T}$. If $\mathcal{T}$ is an arbitrary collection of information structures - that is, with possibly different type set cardinalities - then we say that game form $\Gamma$ is ex post (Nash) consistent for $\mathcal{T}$ if $\left(\Gamma, T, R^{T}\right)$ has an EPE for every $T \in \mathcal{T}$ and every $R^{T} \in W^{T}$. Specifically, if $\mathcal{T}=\{T\}$ with $\left|T^{i}\right|=1$ for every $i \in N$, then an ex post consistent game form $\Gamma$ is Nash consistent (cf. Peleg et al., 2002). We 
say that effectivity function $E$ has an ex post consistent representation for a collection $\mathcal{T}$ of information structures if there exists a game form $\Gamma$ such that $\Gamma$ is a representation of $E$ and $\Gamma$ is ex post consistent for $\mathcal{T}$. We say that $E$ has an ex post consistent representation if there exists a game form $\Gamma$ such that $\Gamma$ represents $E$ and $\Gamma$ is ex post consistent for every information structure $T$.

Lemma 2.5. Suppose $s=\left(s^{1}, \ldots, s^{n}\right) \in S^{N}$ is an $\operatorname{EPE}$ of $\left(\Gamma, T, R^{T}\right)$. Then $B\left(s^{-i}\left(t^{-i}\right)\right) \subseteq L\left(\pi(s(t)), R^{t^{i}}\right)$ for all $t \in T$.

Proof. Let $t \in T^{N}$. Let $a \in B\left(s^{-i}\left(t^{-i}\right)\right)$. Then there is $\sigma^{i} \in \Sigma^{i}$ such that $\pi\left(s^{-i}\left(t^{-i}\right), \sigma^{i}\right)=a$. Hence $\pi(s(t)) R^{t^{i}} a$, since $s$ is an EPE. Therefore $a \in L\left(\pi(s(t)), R^{t^{i}}\right)$.

Remark 2.6. If $s \in S^{N}$ is an EPE of $\left(\Gamma, T, R^{T}\right)$, then Lemma 2.5 and monotonicity imply $L\left(\pi(s(t)), R^{t^{i}}\right) \in E^{\Gamma}(N \backslash\{i\})$ for every $i \in N$ and every $t^{i} \in T^{i}$.

Notation In the sequel, instead of $E(\{i\})$, we usually write $E(i)$.

\section{A representing game form}

In this section we present the basic game form which is used throughout the paper. Later, we will extend and modify this game form in several ways. Alternative representing game forms can be found in many places, notably Peleg (1998), Peleg et al. (2002), Peleg and Peters (2010) and Boros et al. (2010).

Let $E$ be a monotonic and superadditive effectivity function. We fix a numbering of the alternatives in $A$, say $A=\left\{a_{1}, \ldots, a_{|A|}\right\}$. For $i \in N$ let $F^{i}=\{(S, B) \mid i \in S \subseteq N$ and $B \in E(S)\}$. We define the game form $G_{0}=\left(\Sigma^{1}, \ldots, \Sigma^{n} ; \pi ; A\right)$ as follows. The set of actions of $i \in N$ is the set $\Sigma^{i}=\left\{(f, k) \mid f \in F^{i}\right.$ and $\left.k \in\{1, \ldots,|A|\}\right\}$.

Let $\sigma=\left(\sigma^{1}, \ldots, \sigma^{n}\right) \in \Sigma^{N}=\prod_{i \in N} \Sigma^{i}$, where $\sigma^{i}=\left(f^{i}, k^{i}\right) \in \Sigma^{i}$ for $i \in N$. We say that coalition $S \in P_{0}(N)$ is formed if there is $B \in E(S)$ such that $f^{i}=(S, B)$ for all $i \in S$. Define $D \in P_{0}(A)$ as follows. If no coalition is formed, then $D=A$. Otherwise, let $\left\{S_{1}, \ldots, S_{r}\right\}$ be the collection of formed coalitions. For each $j \in\{1, \ldots, r\}$, there is $B_{j} \in E\left(S_{j}\right)$ such that $f^{i}=\left(S_{j}, B_{j}\right)$ for all $i \in S_{j}$. Then let $D=\bigcap_{j=1}^{r} B_{j}$. Note that $D \neq \emptyset$ because $E$ is superadditive and formed coalitions are pairwise disjoint. Suppose $D=\left\{a_{j_{1}}, \ldots, a_{j_{|D|}}\right\}$ with $j_{1}<\ldots<j_{|D|}$. We define $\pi(\sigma)=a_{j_{p}}$, where $p=\sum_{i \in N} k^{i}(\bmod |D|)+1$. 
Proposition 3.1. $G_{0}$ is a representation of $E$.

Proof. We first show that $E(S) \subseteq E^{G_{0}}(S)$ for all $S \in P_{0}(N)$. Let $S \in P_{0}(N)$ and $B \in E(S)$. Choose $\sigma^{i}=((S, B), 1)$ for all $i \in S$. Then the coalition $S$ is formed and hence by definition of $\pi, \pi\left(\sigma^{S}, \tau^{N \backslash S}\right) \in B$ for all $\tau^{N \backslash S} \in \Sigma^{N \backslash S}$. So, $B\left(\sigma^{S}\right) \subseteq B$.

In order to prove the converse inclusion, let $S \in P_{0}(N)$ and $B \in E^{G_{0}}(S)$. Since $E(N)=P_{0}(A)$ and $A \in E(S)$ for all $S \in P_{0}(N)$, we can assume that $S \neq N$ and $B \neq A$. We show that $B \in E(S)$. Let $\sigma^{S} \in \Sigma^{S}$ be such that $B\left(\sigma^{S}\right) \subseteq B$ and for each $i \in N \backslash S$ choose $\tau^{i}$ such that $f^{i}=(N \backslash S, A)$. Consider the action profile $\left(\sigma^{S}, \tau^{N \backslash S}\right)$. In this profile, since $B \neq A, S$ contains at least one formed coalition. Let $\left\{S_{1}, \ldots, S_{r}\right\}$ denote the set of all formed coalitions within $S$; hence, for each $j=1, \ldots, r$ and $i \in S_{j}$, $\sigma^{i}=\left(f^{i}, k^{i}\right)$ with $f^{i}=\left(S_{j}, B_{j}\right)$ for some $B_{j} \in E\left(S_{j}\right)$. Thus, for $\left(\sigma^{S}, \tau^{N \backslash S}\right)$, the set of formed coalitions is $\left\{S_{1}, \ldots, S_{r}, N \backslash \bigcup_{j=1}^{r} S_{j}\right\}$. Since $B_{j} \in E\left(S_{j}\right)$ for all $j \in\{1, \ldots, r\}$, and $\bigcup_{j=1}^{r} S_{j} \subseteq S$, superadditivity and monotonicity imply $\bigcap_{j \in J} B_{j} \in E(S)$. It remains to show that $\bigcap_{j \in J} B_{j} \subseteq B\left(\sigma^{S}\right)$, since then monotonicity implies that $B \in E(S)$.

Take $a \in \bigcap_{j \in J} B_{j}$. Since $S \neq N$, given $\sum_{i \in S} k^{i}, N \backslash S$ is able to choose $\sum_{i \in N \backslash S} k^{i}$ such that $\pi\left(\sigma^{S}, \tau^{N \backslash S}\right)=a$. Hence $a \in B\left(\sigma^{S}\right)$ for every $a \in \bigcap_{j \in J} B_{j}$, which completes the proof.

\section{Two-person effectivity functions}

Throughout this section we assume that $N=\{1,2\}$. The following result is from Peleg et al. (2002), Remark 3.13.

Lemma 4.1. Let $E$ be a superadditive and monotonic effectivity function. The following statements are equivalent:

(i) E has a Nash consistent representation.

(ii) E is maximal.

\subsection{One-sided incomplete information}

In this subsection, we consider the case of one-sided incomplete information. Without loss of generality, we assume that $\left|T^{1}\right|=1$. This means that the preference ordering of one player is commonly known, while the other player possibly has multiple types. 
The main theorem of this subsection shows that in case of one-sided incomplete information, ex post consistent representation imposes no further conditions on the effectivity function compared to Nash consistent representation.

Theorem 4.2. Let $E$ be a superadditive and monotonic effectivity function. Let $\mathcal{T}=\left\{\left(T^{1}, T^{2}\right)|| T^{1} \mid=1\right\}$. The following statements are equivalent:

(i) E has a Nash consistent representation.

(ii) E has an ex post consistent representation for $\mathcal{T}$.

Proof. To prove the implication $(i) \Rightarrow(i i)$, suppose $E$ has a Nash consistent representation, and let $T \in \mathcal{T}$. Then $E$ is maximal by Lemma 4.1. We consider the representing game form $G_{0}$ from Section 3 and show that this game form always contains an EPE. Let $R^{1} \in W$ be the preference ordering of player 1 , and write $a_{1} R^{1} \ldots R^{1} a_{m}$, where $m=|A|$. Let $j \in\{1, \ldots, m\}$ such that $L\left(a_{j}, R^{1}\right) \in E^{\Gamma}(2)$ and $L\left(a_{j+1}, R^{1}\right) \notin E^{\Gamma}(2)$ (where we define $\left.L\left(a_{m+1}, R^{1}\right)=\emptyset\right)$. Define $B_{1}=\left\{a_{1}, \ldots, a_{j}\right\}$. By Proposition 2.1(iii), $B_{1} \in$ $E^{\Gamma}(1)$. Consider the strategy profile where player 1 plays $\sigma^{1}=\left(\left(\{1\}, B_{1}\right), 1\right)$, and every type $t^{2} \in T^{2}$ plays $\sigma^{2}=\left(\left(\{2\}, L\left(a, R^{1}\right)\right), k\right)$, with $a \in B_{1}$ such that $a R^{t^{2}} b$ for all $b \in B_{1}$ and with $k$ such that $\pi\left(\sigma^{1}, \sigma^{2}\right)=a$. It is straightforward to check that this strategy profile is an EPE.

To prove the implication $(i i) \Rightarrow(i)$, suppose $E$ has an ex post consistent representation $\Gamma$ for $\mathcal{T}$. In particular, the game form $\Gamma$ has an ex post equilibrium if all types of player 2 have the same preference ordering. This is equivalent to saying that $E$ has a Nash consistent representation.

\subsection{Two-sided incomplete information}

In this subsection, still for two players, we consider the case of two-sided incomplete information.

A player $i \in N$ is a singleton player if all minimal sets of player $i$ in $E(i)$ are singletons.

Example 4.3. Let $A=\{a, b\}$ with $a \neq b$. There are two possible maximal effectivity functions (if we omit effectivity functions obtainable from these two by permutations of players or alternatives):

(i) $E(1)=E(\{1,2\})=\{\{a\},\{b\}, A\}$ and $E(2)=\{A\}$

(ii) $E(1)=E(2)=\{\{a\}, A\}$ and $E(\{1,2\})=\{\{a\},\{b\}, A\}$ 
Note that in (i) player 1 is a 'dictator'. Also, both effectivity functions contain a singleton player.

The following theorem characterizes all two-player effectivity functions with an ex post consistent representation for all information structures in which both players have at least two types. In fact, its proof shows that if there exists an ex post consistent representation of $E$, then any representation is ex post consistent.

Theorem 4.4. Let $E$ be a maximal effectivity function. Let $\mathcal{T}=\left\{\left(T^{1}, T^{2}\right) \mid\right.$ $\left.\left|T^{1}\right|,\left|T^{2}\right| \geq 2\right\}$. The following statements are equivalent:

(i) E has an ex post consistent representation for $\mathcal{T}$.

(ii) E contains a singleton player.

Proof. To prove the implication $(i) \Rightarrow(i i)$, suppose $E$ does not contain a singleton player. We will present a preference profile for $T \in \mathcal{T}$ with $\left|T^{1}\right|=\left|T^{2}\right|=2$ such that no EPE exists in any representing game form.

Let $B_{1}$ be minimal for player 1 with $\left|B_{1}\right| \geq 2$ and let $B_{2}$ be minimal for player 2 with $\left|B_{2}\right| \geq 2$ (these sets exist, since there is no singleton player). Take $a \in B_{1} \cap B_{2} \neq \emptyset$ (this is possible by superadditivity), and $b \in B_{1}$ with $b \neq a$ (this is possible since $\left|B_{1}\right| \geq 2$ ). We can assume that $|A| \geq 3$, because if $|A|=2$ then every maximal effectivity function contains a singleton player (see Example 4.3). Consider the following preference profile:

\begin{tabular}{ccccc}
\multicolumn{2}{c}{ pl. 1} & & \multicolumn{2}{c}{ pl. 2} \\
$R^{t^{1,1}}$ & $R^{t^{1,2}}$ & & $R^{t^{2,1}}$ & $R^{t^{2,2}}$ \\
\cline { 1 - 2 } \cline { 4 - 5 }$\backslash \backslash\{a, b\}$ & $b$ & $A \backslash B_{1}$ & $A \backslash B_{1}$ \\
$a$ & $A \backslash B_{1}$ & & $b$ & $a$ \\
$b$ & $B_{1} \backslash\{a, b\}$ & & $a$ & $b$ \\
$A \backslash B_{1}$ & $a$ & & $B_{1} \backslash\{a, b\}$ & $B_{1} \backslash\{a, b\}$
\end{tabular}

Now let $\Gamma$ be a representing game form and suppose that $\left(s^{1}, s^{2}\right)$ is an $\mathrm{EPE}$ in the game with this preference profile. We derive a contradiction.

(a) Claim: $b \in B\left(s^{2}\left(t^{2,1}\right)\right)$.

Proof of claim: Since $B_{1} \backslash\{b\} \notin E^{\Gamma}(1)$ ( $B_{1}$ is minimal for player 1), Remark 2.6 implies $\pi\left(s^{1}\left(t^{1,1}\right), s^{2}\left(t^{2,1}\right)\right) \notin B_{1} \backslash\{b\}$. Since $A \backslash B_{1} \notin E^{\Gamma}(2)$ (by superadditivity), Remark 2.6 implies $\pi\left(s^{1}\left(t^{1,1}\right), s^{2}\left(t^{2,1}\right)\right) \notin A \backslash B_{1}$. Hence $\pi\left(s^{1}\left(t^{1,1}\right), s^{2}\left(t^{2,1}\right)\right)=b$, which implies that $b \in B\left(s^{2}\left(t^{2,1}\right)\right)$. 
(b) Claim: $B\left(s^{1}\left(t^{1,2}\right)\right)=B_{1}$.

Proof of claim: Since $b \in B\left(s^{2}\left(t^{2,1}\right)\right)$ by the previous claim, and $b$ is $t^{1,2}$ 's most preferred element, $\pi\left(s^{1}\left(t^{1,2}\right), s^{2}\left(t^{2,1}\right)\right)=b$. This implies, by Lemma 2.5 and minimality of $B_{1}$, that $B\left(s^{1}\left(t^{1,2}\right)\right)=B_{1}$.

Now, since $B\left(s^{1}\left(t^{1,2}\right)\right)=B_{1}$ and $a$ is $t^{2,2}$ 's most preferred alternative from $B_{1}, \pi\left(s^{1}\left(t^{1,2}\right), s^{2}\left(t^{2,2}\right)\right)=a$. Hence, by Remark 2.6, $\{a\}=$ $L\left(\pi\left(s^{1}\left(t^{1,2}\right), s^{2}\left(t^{2,2}\right)\right), R^{t^{1,2}}\right) \in E^{\Gamma}(2)$. Since $a \in B_{2}$, this contradicts minimality of $B_{2}$.

In order to prove the implication $(i i) \Rightarrow(i)$, assume that $E$ contains a singleton player, and let $T \in \mathcal{T}$. We show that for any representing game form $\Gamma$ and any $R^{T} \in W^{T}$, the game $\left(\Gamma, T, R^{T}\right)$ has an EPE. Without loss of generality assume that player 1 is a singleton player. Let $B_{2}=\{a \in A \mid$ $\{a\}$ is a minimal set for player 1$\}$. By Lemma 2.1(ii), we have $B_{2} \in E(2)$. Consider the strategy profile in which every type $t^{1} \in T^{1}$ plays an action $\sigma^{1} \in \Sigma^{1}$ such that $B\left(\sigma^{1}\right)=\{a\}$ for $a \in B_{2}$ such that $a R^{t^{1}} b$ for all $b \in B_{2}$; and in which every type $t^{2} \in T^{2}$ plays an action $\sigma^{2} \in \Sigma^{2}$ such that $B\left(\sigma^{2}\right)=B_{2}$. It is easy to see that this strategy profile is an EPE.

\section{More than two players}

Throughout this section, unless stated otherwise, we assume that $|N| \geq 3$. The following result is from Peleg et al. (2002), Remark 3.11.

Lemma 5.1. Let $E$ be a superadditive and monotonic effectivity function. The following statements are equivalent:

(i) E has a Nash consistent representation.

(ii) $B_{i} \notin E(N \backslash\{i\})$ for all $i \in N \Rightarrow A \backslash \bigcup_{i=1}^{n} B_{i} \neq \emptyset$.

\subsection{One-sided incomplete information}

In this subsection, we assume one-sided incomplete information. The main theorem of this subsection shows that in case of one-sided incomplete information, ex post consistent representation imposes no further conditions on the effectivity function compared to Nash consistent representation. So Theorem 5.2 extends Theorem 4.2 to $n>2$.

The game form that we introduce consists of two parts: actions that assure representation, and equilibrium actions. 
Theorem 5.2. Let $E$ be a superadditive and monotonic effectivity function. Let $\mathcal{T}=\left\{\left(T^{1}, \ldots, T^{n}\right)|| T^{1} \mid \geq 2\right.$ and $\left|T^{i}\right|=1$ for all $\left.i \neq 1\right\}$. The following statements are equivalent:

(i) E has a Nash consistent representation.

(ii) $E$ has an ex post consistent representation for $\mathcal{T}$.

Proof. To prove the implication $(i) \Rightarrow(i i)$, assume that $E$ has a Nash consistent representation, and let $T \in \mathcal{T}$. By Lemma 5.1, if $B_{i} \notin E(N \backslash\{i\})$ for all $i \in N$ then $A \backslash \bigcup_{i=1}^{n} B_{i} \neq \emptyset$.

We extend $G_{0}=\left(\Sigma^{1}, \ldots, \Sigma^{n} ; \pi ; A\right)$ from Section 3 to a game form $G_{1}=$ $\left(\Sigma_{1}^{1}, \ldots, \Sigma_{1}^{n} ; \pi_{1} ; A\right)$, as follows. A selection is a function $\phi: P_{0}(A) \rightarrow A$ such that $\phi(B) \in B$ for every $B \in P_{0}(A)$. Denote by $\Phi$ the set of all selections. The set of actions of $i \in N$ is the set $\Sigma_{1}^{i}=\left\{(f, k, \phi) \mid f \in F^{i} \cup W^{N \backslash\{1\}}, k \in\right.$ $\{1, \ldots,|A|\}$ and $\phi \in \Phi\}$.

Let $\sigma=\left(\sigma^{1}, \ldots, \sigma^{n}\right) \in \Sigma_{1}^{N}$, where $\sigma^{i}=\left(f^{i}, k^{i}, \phi^{i}\right) \in \Sigma_{1}^{i}$ for every $i \in N$. Define $K=\left\{i \in N \mid f^{i} \in F^{i}\right\}$ and $L^{R^{N \backslash\{1\}}}=\left\{i \in N \mid f^{i}=R^{N \backslash\{1\}}\right\}$ for all $R^{N \backslash\{1\}} \in W^{N \backslash\{1\}}$. Note that every $i \in N$ is either in $K$ or in $L^{R^{N \backslash\{1\}}}$ for some $R^{N \backslash\{1\}} \in W^{N \backslash\{1\}}$. In order to define $\pi_{1}(\sigma)$, we consider two cases.

(i) If $\left|L^{R^{N \backslash\{1\}}}\right|<n-1$ for all $R^{N \backslash\{1\}} \in W^{N \backslash\{1\}}$, then we define $\pi_{1}(\sigma)=$ $\pi(\sigma)$, with $\pi$ as in $G_{0}$ (Section 3 ).

(ii) Suppose $\left|L^{R^{N \backslash\{1\}}}\right| \geq n-1$ for some $R^{N \backslash\{1\}} \in W^{N \backslash\{1\}}$. For every $i \neq 1$, we define $B_{i} \notin E(N \backslash\{i\})$ as follows. Let $R^{i}$ be the preference ordering of player $i$ in the profile $R^{N \backslash\{1\}}$, say $a_{1} R^{i} \ldots R^{i} a_{m}$, where $m=|A|$. Choose $j \in\{1, \ldots, m\}$ such that $L\left(a_{j}, R^{i}\right) \in E(N \backslash\{i\})$ and $L\left(a_{j+1}, R^{i}\right) \notin E(N \backslash\{i\})$ (where we define $\left.L\left(a_{m+1}, R^{i}\right)=\emptyset\right)$. Then we define $B_{i}=L\left(a_{j+1}, R^{i}\right)$. Since $B_{i} \notin E(N \backslash\{i\})$ for all $i \neq 1$, we have $A \backslash \bigcup_{i \neq 1} B_{i} \neq \emptyset$. This implies that $C^{R^{N \backslash\{1\}}} \in E(N \backslash\{1\})$, where $C^{R^{N \backslash\{1\}}}=A \backslash \bigcup_{i \neq 1} B_{i}$. To define $\pi_{1}(\sigma)$, we distinguish two further subcases:

(ii.a) If $L^{R^{N \backslash\{1\}}}=N \backslash\{i\}$ with $i \neq 1$, then we define $a=\phi^{1}\left(C^{R^{N \backslash\{1\}}}\right)$, and $\pi_{1}(\sigma)=\phi^{i}\left(L\left(a, R^{i}\right)\right)$.

(ii.b) If $L^{R^{N \backslash\{1\}}}=N \backslash\{1\}$ or $L^{R^{N \backslash\{1\}}}=N$, then we define $\pi_{1}(\sigma)=$ $\phi^{1}\left(C^{R^{N \backslash\{1\}}}\right)$.

Claim: $G_{1}$ is a representation of $E$.

To prove this claim, we first show that $E(S) \subseteq E^{G_{1}}(S)$ for all $S \in P_{0}(N)$. Let $S \in P_{0}(N)$ and $B \in E(S)$. We distinguish three cases. 
- $|S| \geq 2$. Choose $\sigma^{i}=\left((S, B), 1, \phi^{i}\right)$, with $\phi^{i}$ chosen arbitrarily, for all $i \in S$. Then the coalition $S$ is formed and hence by definition of $\pi_{1}$, $\pi_{1}\left(\sigma^{S}, \tau^{N \backslash S}\right)=\pi\left(\sigma^{S}, \tau^{N \backslash S}\right) \in B$ for all $\tau^{N \backslash S} \in \Sigma_{1}^{N \backslash S}$. So, $B\left(\sigma^{S}\right) \subseteq B$.

- $S=\{1\}$. Choose $\sigma^{1}=\left((\{1\}, B), 1, \phi^{1}\right)$, where $\phi^{1}$ is such that $\phi^{1}\left(B^{\prime}\right) \in$ $B$ for all $B^{\prime}$ with $B^{\prime} \cap B \neq \emptyset$.

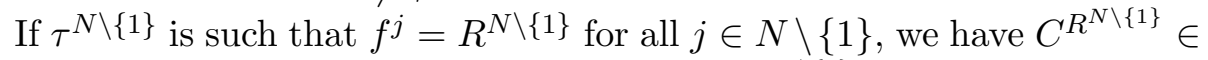
$E(N \backslash\{1\})$ and thus by superadditivity $A \backslash C^{R^{N \backslash\{1\}}} \notin E(1)$. Hence, by monotonicity, $B \cap C^{R^{N \backslash\{1\}}} \neq \emptyset$, which means that $\phi^{1}$ selects an alternative from $B$.

For all other $\tau^{N \backslash\{1\}} \in \Sigma_{1}^{N \backslash\{1\}}$, coalition $\{1\}$ forms. Thus, by definition of $\pi_{1}, \pi_{1}\left(\sigma^{1}, \tau^{N \backslash\{1\}}\right)=\pi\left(\sigma^{1}, \tau^{N \backslash\{1\}}\right) \in B$ for all $\tau^{N \backslash\{1\}} \in \Sigma_{1}^{N \backslash\{1\}}$.

- $S=\{i\}$, with $i \neq 1$. Choose $\sigma^{i}=\left((\{i\}, B), 1, \phi^{i}\right)$, where $\phi^{i}$ is such that $\phi^{i}\left(B^{\prime}\right) \in B$ for all $B^{\prime}$ with $B^{\prime} \cap B \neq \emptyset$.

If $\tau^{N \backslash\{i\}}$ is such that $f^{j}=R^{N \backslash\{1\}}$ for all $j \in N \backslash\{i\}$, the definition of $C^{R^{N \backslash\{1\}}}$ implies $L\left(a, R^{i}\right) \in E(N \backslash\{i\})$ for all $a \in C^{R^{N \backslash\{1\}}}$. Then superadditivity implies that $A \backslash L\left(a, R^{i}\right) \notin E(i)$. Hence $B \cap L\left(a, R^{i}\right) \neq \emptyset$ for all

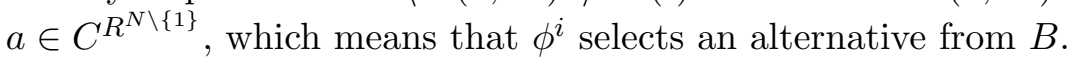

For all other $\tau^{N \backslash\{i\}} \in \Sigma_{1}^{N \backslash\{i\}}$, coalition $\{i\}$ forms. Thus, by definition of $\pi_{1}, \pi_{1}\left(\sigma^{i}, \tau^{N \backslash\{i\}}\right)=\pi\left(\sigma^{i}, \tau^{N \backslash\{i\}}\right) \in B$ for all $\tau^{N \backslash\{i\}} \in \Sigma_{1}^{N \backslash\{i\}}$.

In order to prove the converse inclusion, let $S \in P_{0}(N)$ and $B \in E^{G_{1}}(S)$. Since $E(N)=P_{0}(A)$ and $A \in E(S)$ for all $S \in P_{0}(N)$, we can assume that $S \neq N$ and $B \neq A$. We show that $B \in E(S)$. Let $\sigma^{S} \in \Sigma^{S}$ be such that $B\left(\sigma^{S}\right) \subseteq B$. We only treat the case where $|S|=n-1$ and $\sigma^{S}$ is such that $f^{i}=R^{N \backslash\{1\}}$ for some $R^{N \backslash\{1\}} \in W^{N \backslash\{1\}}$ and for all $i \in S$. For all other cases, it is sufficient to note that $G_{1}$ is an extension of $G_{0}$. We distinguish two subcases.

- $1 \notin S$. Then, by construction of the game form, $B\left(\sigma^{S}\right)=C^{R^{N \backslash\{1\}}}$. By definition of $C^{R^{N \backslash\{1\}}}$, we have $C^{R^{N \backslash\{1\}}} \in E(N \backslash\{1\})=E(S)$ and thus by monotonicity $B \in E(S)$.

- $1 \in S$ and $j \notin S$. Then by construction $B\left(\sigma^{S}\right)=L\left(a, R^{j}\right)$ for some $a \in C^{R^{N \backslash\{1\}}}$. By definition of $C^{R^{N \backslash\{1\}}}$ and since $j \neq 1$, we have $L\left(a, R^{j}\right) \in$ $E(N \backslash\{j\})=E(S)$ for all $a \in C^{R^{N \backslash\{1\}}}$ and thus, by monotonicity, $B \in E(S)$.

This concludes the proof of the Claim.

We now prove that $G_{1}$ is ex post consistent for $T$. Let $R^{T} \in W^{T}$. Consider the following strategy profile $s \in S^{N}$. For every $t^{i} \in T^{i}$ with $i \neq 1, s\left(t^{i}\right)=\left(R^{T^{-1}}, 1, \phi^{i}\right)$, with $\phi^{i}$ chosen arbitrarily. For every $t^{1} \in T^{1}$, 
$s\left(t^{1}\right)=\left(R^{T^{-1}}, 1, \phi^{1}\right)$ is such that $\phi^{1}$ selects alternative $a \in C^{R^{N \backslash\{1\}}}$, where $a R^{t^{1}} b$ for all $b \in C^{R^{N \backslash\{1\}}}$.

If $t^{1} \in T^{1}$ deviates, $t^{1}$ is able to choose an alternative from $C^{R^{N \backslash\{1\}}}$. Since for $a \in C^{R^{N \backslash\{1\}}}$ we have $a R^{t^{1}} b$ for all $b \in C^{R^{N \backslash\{1\}}}, t^{1}$ is not able to improve. If $t^{i} \in T^{i}$, with $i \neq 1$, deviates, $t^{i}$ is able to choose from $L\left(a, R^{t^{i}}\right)$. Since $a R^{t^{i}} b$ for all $b \in L\left(a, R^{t^{i}}\right), t^{i}$ is not able to improve. Hence this profile is an EPE.

For the implication $(i i) \Rightarrow(i)$, assume that $E$ has an ex post consistent representation $\Gamma$ for $\mathcal{T}$. In particular, the game form $\Gamma$ should contain an ex post equilibrium if all types of player 1 have the same preference ordering. This is equivalent to saying that $E$ has a Nash consistent representation.

\subsection{General incomplete information}

In this subsection, we characterize effectivity functions that have an ex post consistent representation for any number of types. The next theorem extends Theorem 4.4 to $n>2$.

Theorem 5.3. Let $E$ be a superadditive and monotonic effectivity function. The following statements are equivalent:

(i) E has an ex post consistent representation.

(ii) There exists an $i \in N$ and $C \in E(N \backslash\{i\})$ such that $\{a\} \in E(N \backslash\{j\})$ for all $a \in C$ and for every $j \neq i$.

Proof. We first prove the implication $(i) \Rightarrow(i i)$. We assume that for every $i \in N$ and every $B_{i} \in E(N \backslash\{i\})$, there is $a \in B_{i}$ such that $\{a\} \notin E(N \backslash\{j\})$ for some $j \neq i$. First, we define a specific preference profile. Second, we show that for this particular preference profile no EPE exists in any representing game form.

We define the sets $A_{1, i}=\{a \in A \mid\{a\} \notin E(N \backslash\{i\})$ and $\{a\} \in$ $E(N \backslash\{j\})$ for all $j \neq i\}$ for all $i \in N$, and $A_{2}=\{a \in A \mid$ there are $i, j \in$ $N$, with $i \neq j$, such that $\{a\} \notin E(N \backslash\{i\})$ and $\{a\} \notin E(N \backslash\{j\})\}$. By our assumption, $\bigcup_{i=1}^{n} A_{1, i} \cup A_{2}=A$. Moreover, $A_{1, i} \cap A_{2}=\emptyset$ for all $i \in N$ and $A_{1, i} \cap A_{1, j}=\emptyset$ for all $i \neq j$.

The definition of the announced preference profile will be given by an algorithm, as follows. We start with an information structure $\left(T^{1}, \ldots, T^{n}\right)$ such that $\left|T^{i}\right|=1$ for all $i \in N$. Let $t=\left(t^{1}, \ldots, t^{n}\right) \in T^{1} \times \cdots \times T^{n}$ denote the corresponding type vector. In the algorithm, for every $i \in N$, two sets $H_{i} \subseteq A$ and $L_{i} \subseteq A$ are defined, and a preference ordering $R^{t^{i}}$ 
over $H_{i} \cup L_{i}$ such that for all $a \in H_{i}$ and all $b \in L_{i}, a P^{t^{i}} b$ (alternatives in $H_{i}$ are strictly preferred to alternatives in $L_{i}$ ). We iteratively extend this preference ordering by adding an $a \in A$ to either $H_{i}$ or $L_{i}$ for every $i \in N$, until $H_{i} \cup L_{i}=A$. Under a specific condition, extra types may be added. See Algorithm 1. ${ }^{2}$

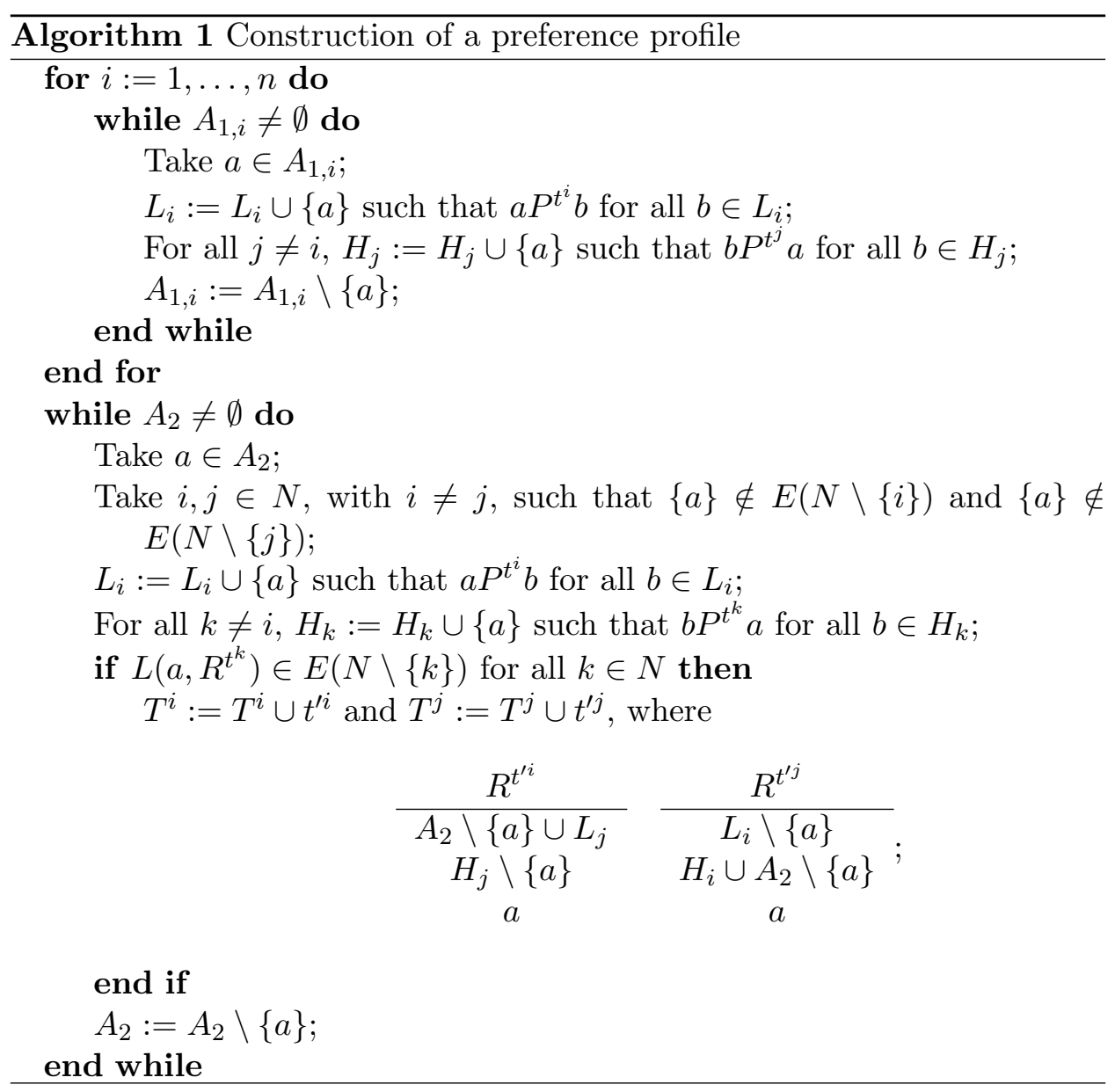

Consider the constructed preference profile $R^{T}$ with associated information structure $T$ and let $\Gamma$ be a representing game form. Suppose that $\left(s^{1}, \ldots, s^{n}\right)$ is an EPE in $\left(\Gamma, T, R^{T}\right)$. We derive a contradiction.

Let $i \in N$. Since for all $a \in A_{1, i},\{a\} \in E^{\Gamma}(N \backslash\{j\})$ for all $j \neq i$, our initial assumption implies $A_{1, i} \notin E^{\Gamma}(N \backslash\{i\})$. Thus, for all $i \in N$ and for all

\footnotetext{
${ }^{2}$ See Example 5.6 for an illustration of this algorithm. The constructed preference profile can also be defined directly, but the description via the algorithm is more transparent.
} 
$a \in A_{1, i}$, we have $L\left(a, R^{t^{i}}\right) \notin E^{\Gamma}(N \backslash\{i\})$. Hence, by Remark 2.6, we have $\pi(s(t)) \notin \bigcup_{i=1}^{n} A_{1, i}$ and thus $\pi(s(t)) \in A_{2}$ with $L\left(\pi(s(t)), R^{t^{i}}\right) \in E^{\Gamma}(N \backslash\{i\})$ for all $i \in N$.

Let $a=\pi(s(t))$. If no new types were added in the algorithm, then there was a player $k \in N$ such that $L\left(a, R^{t^{k}}\right)=L\left(\pi(s(t)), R^{t^{k}}\right) \notin E(N \backslash\{k\})$, but this contradicts Remark 2.6. So we may assume that there are $i, j \in N$, with $i \neq j$, be such that $t^{\prime i}$ and $t^{\prime j}$ are added for $a$. In the step of the algorithm where $t^{i}$ and $t^{\prime j}$ are added, $a$ becomes the most preferred element of $L_{i}$ for $t^{i}$ and the least preferred element of $H_{j}$ for $t^{j}$. Since each remaining alternative $b \in A_{2} \backslash\{a\}$ becomes either the new best alternative of $L_{i}$ or $L_{j}$ or the new worst alternative of $H_{i}$ or $H_{j}$, we have at the end of the algorithm $b P^{t^{i}} a$ and $a P^{t^{j}} b$ for all remaining $b \in A_{2} \backslash\{a\}$. So in fact, the preference orderings of $t^{\prime i}$ and $t^{\prime j}$ are as follows:

$$
\begin{aligned}
& \frac{R^{t^{i}}}{L\left(a, R^{t^{j}}\right) \backslash\{a\}} \frac{R^{t^{j}}}{L\left(a, R^{t^{2}}\right) \backslash\{a\}} \\
& A \backslash L\left(a, R^{t^{j}}\right) \quad A \backslash L\left(a, R^{t^{i}}\right) \\
& \text { a } a
\end{aligned}
$$

Observe the following:

(a) Lemma 2.5 implies that $B\left(s^{-i}\left(t^{-i}\right)\right) \subseteq L\left(a, R^{t^{i}}\right)$. Since $L\left(a, R^{t^{i}}\right) \in$ $E(N \backslash\{i\})$ by the condition for adding types $t^{\prime i}$ and $t^{\prime j}$ in the algorithm, and $\{a\} \notin E(N \backslash\{i\})$ since $a \in A_{2}$, we have $L\left(a, R^{t^{i}}\right) \backslash\{a\} \neq \emptyset$. Since $a$ is $t^{\prime i}$ 's least preferred element, we have $\pi\left(s^{-i}\left(t^{-i}\right), s^{i}\left(t^{\prime i}\right)\right) \in$ $L\left(a, R^{t^{i}}\right) \backslash\{a\}$. But this implies that $\pi\left(s^{-i, j}\left(t^{-i, j}\right), s^{i}\left(t^{\prime i}\right), s^{j}\left(t^{\prime j}\right)\right) \neq a$ and $\pi\left(s^{-i, j}\left(t^{-i, j}\right), s^{i}\left(t^{\prime i}\right), s^{j}\left(t^{\prime j}\right)\right) \notin A \backslash L\left(a, R^{t^{i}}\right) ! \underline{3}$

(b) Lemma 2.5 implies that $B\left(s^{-j}\left(t^{-j}\right)\right) \subseteq L\left(a, R^{t^{j}}\right)$. Similarly as in (a), we have $L\left(a, R^{t^{j}}\right) \backslash\{a\} \neq \emptyset$. Since $a$ is $t^{\prime j}$ 's least preferred element, we have $\pi\left(s^{-j}\left(t^{-j}\right), s^{j}\left(t^{\prime j}\right)\right) \in L\left(a, R^{t^{j}}\right) \backslash\{a\}$. But this implies that $\pi\left(s^{-i, j}\left(t^{-i, j}\right), s^{i}\left(t^{\prime i}\right), s^{j}\left(t^{\prime j}\right)\right) \notin A \backslash L\left(a, R^{t^{j}}\right)$.

If we show that $\{a\} \cup A \backslash L\left(a, R^{t^{i}}\right) \cup A \backslash L\left(a, R^{t^{j}}\right)=A$, then we have proved that there is no Nash outcome for $\left(t^{-i, j}, t^{\prime i}, t^{\prime j}\right)$, which is in contradiction with the assumption that $\left(s^{1}, \ldots, s^{n}\right)$ is an EPE. Note that it is sufficient to show that $L\left(a, R^{t^{i}}\right) \backslash\{a\} \subseteq A \backslash L\left(a, R^{t^{j}}\right)$.

Take $b \in L\left(a, R^{t^{i}}\right)$, with $b \neq a$. Since $a P^{t^{i}} b$, in the algorithm $b$ became the new best element of $L_{i}$ for $t^{i}$ before $a$ became the new best element of $L_{i}$

\footnotetext{
${ }^{3}$ We define $s^{-i, j}\left(t^{-i, j}\right)$ as the vector $\left(s^{k}\left(t^{k}\right)\right)_{k \neq i, j}$
} 
for $t^{i}$. So also, $b$ became the new worst element of $H_{j}$ for $t^{j}$ before $a$ became the new worst element of $H_{j}$ for $t^{j}$. Hence $b P^{t^{j}} a$ and thus $b \in A \backslash L\left(a, R^{t^{j}}\right)$. This concludes the proof of the implication $(i) \Rightarrow(i i)$.

We now prove the implication $(i i) \Rightarrow(i)$. We assume that there exists an $i \in N$ and $C \in E(N \backslash\{i\})$ such that $\{a\} \in E(N \backslash\{j\})$ for all $a \in C$ and for every $j \neq i$. W.l.o.g. assume $i=1$. We write $C=\left\{a_{1}, \ldots, a_{|C|}\right\}$.

We extend $G_{0}=\left(\Sigma^{1}, \ldots, \Sigma^{n} ; \pi ; A\right)$ from Section 3 to a game form $G_{2}=$ $\left(\Sigma_{2}^{1}, \ldots, \Sigma_{2}^{n} ; \pi_{2} ; A\right)$ as follows. The set of actions of $i \in N$ is the set $\Sigma_{2}^{i}=$ $\left\{(f, k) \mid f \in F^{i} \cup\{v\}\right.$ and $\left.k \in\{1, \ldots,|A|\}\right\}$.

Let $\sigma=\left(\sigma^{1}, \ldots, \sigma^{n}\right) \in \Sigma_{2}^{N}$, where $\sigma^{i}=\left(f^{i}, k^{i}\right) \in \Sigma_{2}^{i}$ for every $i \in N$. Define $K=\left\{i \in N \mid f^{i} \in F^{i}\right\}$ and $L=\left\{i \in N \mid f^{i}=v\right\}$. Then $N=K \cup L$ and $K \cap L=\emptyset$. To define $\pi_{2}(\sigma)$, we distinguish two cases:

(i) $|L|<n-1$. Then $\pi_{2}(\sigma)=\pi(\sigma)$, with $\pi$ the outcome function of $G_{0}$ (see Section 3).

(ii) $|L| \geq n-1$. Then $\pi_{2}(\sigma)=a_{p} \in C$, where $p=k^{1}(\bmod |C|)+1$.

Claim: $G_{2}$ is a representation of $E$.

To prove this claim we first show that $E(S) \subseteq E^{G_{2}}(S)$ for all $S \in P_{0}(N)$. Let $S \in P_{0}(N)$ and $B \in E(S)$. We distinguish three cases

- $|S| \geq 2$. Choose $\sigma^{i}=((S, B), 1)$ for all $i \in S$. Then coalition $S$ is formed and hence by definition of $\pi_{2}, \pi_{2}\left(\sigma^{S}, \tau^{N \backslash S}\right) \in B$ for all $\tau^{N \backslash S} \in \Sigma_{2}^{N \backslash S}$. So, $B\left(\sigma^{S}\right) \subseteq B$.

- $S=\{1\}$. Choose $\sigma^{1}=\left((\{1\}, B), k^{1}\right)$, where $k^{1}$ is defined as follows. Since $C \in E(N \backslash\{1\})$, superadditivity implies that $A \backslash C \notin E(1)$ and thus $B \cap C \neq \emptyset$. This implies that we can choose $k^{1}$ such that $\pi_{2}\left(\sigma^{1}, \tau^{N \backslash\{1\}}\right) \in B$ if $\tau^{N \backslash\{1\}}$ is such that $f^{j}=v$ for all $j \in N \backslash\{1\}$. For all other $\tau^{N \backslash\{1\}} \in \Sigma_{2}^{N \backslash\{1\}}$, it is sufficient to note that coalition $\{1\}$ forms.

- $S=\{i\}$, with $i \neq 1$. Choose $\sigma^{i}=((\{i\}, B), 1)$. Since $\{a\} \in E(N \backslash\{i\})$ for all $a \in C$, superadditivity implies that $B \supseteq C$. Thus, by definition of $\pi_{2}, \pi_{2}\left(\sigma^{i}, \tau^{N \backslash\{i\}}\right) \in B$ for all $\tau^{N \backslash\{i\}} \in \Sigma_{2}^{N \backslash\{i\}}$.

In order to prove the converse inclusion, let $S \in P_{0}(N)$ and $B \in E^{G_{2}}(S)$. We show that $B \in E(S)$. Let $\sigma^{S} \in \Sigma^{S}$ such that $B\left(\sigma^{S}\right) \subseteq B$. We only treat the case where $|S|=n-1$ and $\sigma^{S}$ is such that $f^{i}=v$ for all $i \in S$. For all other cases, it is sufficient to note that $G_{2}$ is an extension of $G_{0}$. We distinguish two cases. 
- $1 \notin S$. Then, by construction of the game form, $B\left(\sigma^{S}\right)=C$. By assumption, $C \in E(N \backslash\{1\})=E(S)$ and thus by monotonicity $B \in E(S)$.

- $1 \in S$ and $j \notin S$. Then by construction $B\left(\sigma^{S}\right)=\{a\}$ for some $a \in C$. By assumption, $\{a\} \in E(N \backslash\{j\})=E(S)$, and thus by monotonicity $B \in E(S)$.

This concludes the proof of the claim.

Now let $T$ be an arbitrary information structure. It remains to prove that $G_{2}$ is ex post consistent for $T$. Let $R^{T} \in W^{T}$. Consider the following strategy profile $s \in S^{N}$. For every $t^{i} \in T^{i}$, with $i \neq 1, s\left(t^{i}\right)=(v, 1)$. For every $t^{1} \in T^{1}, s\left(t^{1}\right)=\left(v, k^{1}\right)$ such that $k^{1}$ selects alternative $a \in C$, where $a R^{t^{1}} b$ for all $b \in C$.

If $t^{1} \in T^{1}$ deviates, $t^{1}$ is able to choose an alternative from $C$. Since $a R^{t^{1}} b$ for all $b \in C, t^{1}$ is not able to improve. If $t^{i} \in T^{i}$, with $i \neq 1$, deviates, the outcome does not change. Hence this profile is an EPE.

Remark 5.4. It is not difficult to verify that Condition (ii) of Theorem 5.3 implies condition (ii) of Lemma 5.1 .

Corollary 5.5. Let $E$ be a superadditive and monotonic effectivity function with a Nash consistent representation. If $E(N \backslash\{i\})=\{A\}$ for some $i \in N$, then $E$ has an ex post consistent representation.

Proof. If $E(N \backslash\{i\})=\{A\}$ for some $i \in N$, then Lemma 5.1 (ii) implies that $E(N \backslash\{j\})=P_{0}(A)$ for all $j \neq i$. By Theorem 5.3, $E$ has an ex post consistent representation.

Example 5.6. Let $N=\{1,2,3\}$ and $A=\{a, b, c, d, e\}$. Let $E$ be a superadditive and monotonic effectivity function with

$$
\begin{aligned}
& E(\{1,2\})=\{\{c\},\{d\},\{e\},\{a, b\}\}^{+} \\
& E(\{1,3\})=\{\{a\},\{b\},\{e\},\{c, d\}\}^{+} \\
& E(\{2,3\})=\{\{a\},\{c\},\{b, d\},\{b, e\},\{d, e\}\}^{+} .
\end{aligned}
$$

(The superscript + indicates that all supersets are included as well.) We illustrate the procedure of Algorithm 1. We have $A_{1,1}=\{e\}, A_{1,2}=\{c\}$, $A_{1,3}=\{a\}$ and $A_{2}=\{b, d\}$. After the first while loop, we have the following 
preference profile:

$$
\begin{aligned}
& \frac{R^{t^{1}}}{c} \frac{R^{t^{2}}}{e} \frac{R^{t^{3}}}{e} \\
& \begin{array}{lll}
a & a & c
\end{array} \\
& \begin{array}{lll}
a & \ldots
\end{array} \\
& \begin{array}{ccc}
\cdots & \cdots & \cdots \\
e & c & a
\end{array}
\end{aligned}
$$

\begin{tabular}{|c|c|c|c|c|c|c|}
\hline$R^{t^{1}}$ & $R^{t^{\prime 1}}$ & $R^{t^{\prime \prime 1}}$ & $R^{t^{2}}$ & $R^{t^{\prime 2}}$ & $R^{t^{3}}$ & $R^{t^{\prime 3}}$ \\
\hline$c$ & $a, d$ & $c$ & $e$ & $b, e$ & $e$ & $e$ \\
\hline$a$ & $c, e$ & $a, b, e$ & $a$ & $a, c$ & $c$ & $a, c, d$ \\
\hline$d$ & $b$ & $d$ & $b$ & $d$ & $b$ & $b$ \\
\hline$b$ & & & $d$ & & $d$ & \\
\hline$e$ & & & $c$ & & $a$ & \\
\hline
\end{tabular}

After the second while loop, we have the following preference profile:

This is the profile, used in the proof of Theorem [5.3, for which there is no ex post Nash equilibrium. However, in this example there is another preference profile with less types for which also no representing game form with an EPE exists. This can be shown by using similar arguments (omitted here) as in the proof of Theorem 4.4 .

\begin{tabular}{cccccccc}
$R^{t^{1,1}}$ & $R^{t^{1,2}}$ & & $R^{t^{2,1}}$ & $R^{t^{2,2}}$ & & $R^{t^{3,1}}$ & $R^{t^{3,2}}$ \\
\cline { 1 - 2 } \cline { 6 - 8 }$a$ & $c$ & & $a$ & $d$ & & $e$ & $e$ \\
$d$ & $b$ & & $b$ & $a$ & & $d$ & $a$ \\
$c$ & $e$ & & $e$ & $b$ & & $d$ & $c$ \\
$e$ & $a$ & & $c$ & $e$ & & $b$ & $d$ \\
$b$ & $d$ & & $d$ & $c$ & & $a$ & $b$
\end{tabular}

Thus, the algorithm used in the proof of Theorem 5.3 is not always the most efficient one in terms of the number of types needed in order to obtain nonexistence of an ex post Nash equilibrium.

Example 5.7. Let $N=\{1,2,3\}$ and $A=\{a, b, c\}$. Consider the following effectivity function: $E(1)=E(2)=\{\{a, b\}, A\}, E(3)=\{A\}, E(\{1,2\})=$

\begin{tabular}{|c|c|c|c|c|c|c|}
\hline$M_{1}$ & $L$ & $M$ & $R$ & $M_{2}$ & $L \quad M$ & $R$ \\
\hline$T$ & $a$ & $a$ & $a$ & $T$ & $b$ & $b$ \\
\hline$C$ & $a$ & $b$ & $c$ & $C$ & $b$ & $a$ \\
\hline$B$ & $a$ & $c$ & $b$ & $B$ & $b$ & \\
\hline
\end{tabular}
$\{\{a, b\},\{a, c\},\{b, c\}\}^{+}$and $E(\{1,3\})=E(\{2,3\})=\{\{a\},\{b\}\}^{+}$. By Theorem [5.3, this effectivity function has an ex post consistent representation. Consider the following representation: 
in which $\Sigma^{1}=\{T, C, B\}, \Sigma^{2}=\{L, M, R\}$ and $\Sigma^{3}=\left\{M_{1}, M_{2}\right\}$. This representation is ex post consistent for any $T$. Namely, the following strategy profile always constitutes an EPE: all $t^{1} \in T^{1}$ play $T$, all $t^{2} \in T^{2}$ play $L$ and all $t^{3} \in T^{3}$ with $a R^{t^{3}} b$ play $M_{1}$ and all $t^{3} \in T^{3}$ with $b P^{t^{3}} a$ play $M_{2}$. Observe that $E$ has no 'dictator' (i.e., a player who is effective for every singleton. In the given equilibrium, however, player 3 can act as if he is the dictator over $\{a, b\}$.

\subsection{Maximally two types per player}

Theorem 5.3 characterizes all effectivity functions for which no ex post consistent representation exists, i.e.: there is no game form that represents the effectivity function and that has an ex post Nash equilibrium for any information structure $T=\left(T^{1}, \ldots, T^{n}\right)$ and any profile of preferences for all types. Although it is not difficult to derive an upper bound on the number of types needed (e.g., by considering Algorithm 1), it is less obvious how to derive a sharp upper bound (depending on the effectivity function at hand). From the preceding sections we already know that ex post consistency is implied by Nash consistency for information structures where at most one player has more than type.

In this subsection we provide a few partial answers to this question for other cases. Specifically, the following proposition presents a condition under which at most two types per player are needed to show that no ex post consistent representation exists. Part (a) follows almost directly from Theorem 5.3, the new contribution is part (b), which gives a condition under which it is sufficient to have two players with two types each in order to have nonexistence of an ex post consistent representation, all other players having just one type.

Proposition 5.8. Let E have a Nash consistent representation, but no ex post consistent representation. Then:

(a) There exists an $i \in N$ and a minimal $B_{i} \in E(N \backslash\{i\})$, with $\left|B_{i}\right| \geq 2$, such that there is a $j \neq i$ with $\{a\} \notin E(N \backslash\{j\})$ for an $a \in B_{i}$.

(b) For $i, B_{i}$, a and $j$ as in (a), if either $A \backslash B_{i} \notin E(N \backslash\{j\})$ or $A \backslash B_{i} \in$ $E(N \backslash\{j\})$ is minimal for $N \backslash\{j\}$, with $\left|A \backslash B_{i}\right| \geq 2$, then $E$ has no ex post consistent representation for $\mathcal{T}$, where $\mathcal{T}=\left\{\left(T^{1}, \ldots, T^{n}\right)|| T^{i} \mid=\right.$ $\left|T^{j}\right|=2,\left|T^{k}\right|=1$ for all $\left.k \in N \backslash\{i, j\}\right\}$.

Proof. (a) Suppose, contrary to what we wish to show, that $\left|B_{i}\right|=1$ for all minimal $B_{i} \in E(N \backslash\{i\})$ for all $i \in N$. For all $i \in N$, define $B_{i}=\{a \in$ 
$A \mid\{a\} \notin E(N \backslash\{i\})\}$. Then $B_{i} \notin E(N \backslash\{i\})$ for all $i \in N$. Since $E$ has no ex post consistent representation, Theorem 5.3 implies for all $a \in A$ that $\{a\} \notin E(N \backslash\{i\})$ for some $i \in N$. But then $A \backslash \bigcup_{i=1}^{n} B_{i}=\emptyset$, which contradicts with the assumption that $E$ has a Nash consistent representation (Lemma 5.1).

(b) Let $i, B_{i}, a$ and $j$ be as in $(a)$. Let $T$ satisfy $\left|T^{i}\right|=2,\left|T^{j}\right|=2$ and $\left|T^{k}\right|=1$ for all $k \neq i, j$.

First suppose that $A \backslash B_{i} \notin E(N \backslash\{j\})$. Consider the following preference profile:

\begin{tabular}{ccccc}
\multicolumn{2}{c}{ pl. j } & \multicolumn{2}{c}{ pl. i } \\
$R^{t^{j, 1}}$ & $R^{t^{j, 2}}$ & & $R^{t^{i, 1}}$ & $R^{t^{i, 2}}$ \\
\cline { 1 - 2 } \cline { 4 - 5 }$\backslash\{a, b\}$ & $b$ & $A \backslash B_{i}$ & $A \backslash B_{i}$ \\
$a$ & $A \backslash B_{i}$ & & $b$ & $a$ \\
$b$ & $B_{i} \backslash\{a, b\}$ & & $a$ & $b$ \\
$A \backslash B_{i}$ & $a$ & & $B_{i} \backslash\{a, b\}$ & $B_{i} \backslash\{a, b\}$
\end{tabular}

By using the same argument as in the proof of Theorem 4.4, it follows, similarly as there, that there is no EPE in any representing game form.

Second, suppose that $A \backslash B_{i} \in E(N \backslash\{j\})$ is minimal for $N \backslash\{j\}$ and $\left|A \backslash B_{i}\right| \geq 2$. Define $B_{j}=A \backslash B_{i}$. Since $E$ has no ex post consistent representation, by Theorem 5.3 there is some $c \in B_{j}$ and $k \in N$ with $k \neq j$ such that $\{c\} \notin E(N \backslash\{k\})$. Take $d \in B_{j}$ with $d \neq c$.

If $k=i$, consider the following preference profile:

\begin{tabular}{ccccc}
\multicolumn{2}{c}{ pl. j } & \multicolumn{2}{c}{ pl. i } \\
$R^{t^{j, 1}}$ & $R^{t^{j, 2}}$ & & $R^{t^{i, 1}}$ & $R^{t^{i, 2}}$ \\
\cline { 1 - 2 } \cline { 4 - 5 }$\backslash\{a, b\}$ & $b$ & & $B_{j} \backslash\{c, d\}$ & $d$ \\
$a$ & $B_{i} \backslash\{a, b\}$ & & $c$ & $B_{j} \backslash\{c, d\}$ \\
$b$ & $c$ & & $a$ \\
$d$ & $d$ & & $b$ & $b$ \\
$c$ & $B_{j} \backslash\{c, d\}$ & $a$ & $B_{i} \backslash\{a, b\}$ \\
$B_{j} \backslash\{c, d\}$ & $a$ & $B_{i} \backslash\{a, b\}$ & $c$
\end{tabular}

By using a similar argument as in the proof of Theorem 4.4, it follows that there is no Nash equilibrium outcome between $\left(t^{-i, j}, t^{i, 1}, t^{j, 1}\right)$ in any representing game form, and thus no EPE. 
If $k \neq i$, consider the following preference profile:

\begin{tabular}{|c|c|c|c|c|}
\hline pl. k & $R^{t^{j, 1}}$ & $\mathrm{j} \quad R^{j^{j, 2}}$ & \multicolumn{2}{|c|}{ pl. i } \\
\hline$R^{t^{k}}$ & $B_{i} \backslash\{a, b\}$ & $b$ & $R^{t^{i, 1}}$ & $R^{t^{i, 2}}$ \\
\hline$d$ & a & $B_{i} \backslash\{a, b\}$ & $A \backslash B_{i}$ & $A \backslash B_{i}$ \\
\hline$B_{i}$ & $b$ & $c$ & $b$ & $a$ \\
\hline$B_{j} \backslash\{c, d\}$ & $d$ & $d$ & $a$ & $b$ \\
\hline$c$ & $c$ & $B_{j} \backslash\{c, d\}$ & $B_{i} \backslash\{a, b\}$ & $B_{i} \backslash\{a, b\}$ \\
\hline & $B_{j} \backslash\{c, d\}$ & $a$ & & \\
\hline
\end{tabular}

By using a similar argument as in the proof of Theorem 4.4, it follows that there is no Nash equilibrium outcome between $\left(t^{-i, j, k}, t^{i, 1}, t^{j, 1}, t^{k, 1}\right)$ in any representing game form, and thus no EPE.

We conclude with an example of an effectivity function that has no ex post consistent representation. However, this effectivity function has an ex post consistent representation for two types per player.

Example 5.9. Let $N=\{1,2,3\}$ and $A=\{a, b, c, d, e\}$. Let $E$ be a superadditive and monotonic effectivity function with $E(\{1,2\})=P_{0}(A)$ and $E(\{1,3\})=E(\{2,3\})=\{\{x, y\} \mid x, y \in A, x \neq y\}$. By Theorem 5.3, $E$ has no ex post consistent representation. Let $\mathcal{T}=\left\{\left(T^{1}, T^{2}, T^{3}\right)|| T^{1}|=| T^{2} \mid=\right.$ $\left.\left|T^{3}\right|=2\right\}$. Then $E$ has an ex post consistent representation for $\mathcal{T}$. Such a representation is constructed in the Appendix.

\section{References}

Abdou, J., Keiding, H. (1991) Effectivity functions in social choice. Kluwer Academic Publishers, Dordrecht.

d'Aspremont, C., Peleg, B. (1988) Ordinal Bayesian incentive compatible representations of committees. Social Choice and Welfare 5, 261-279.

Boros, E., Elbassioni, K., Gurvich, V., Makino, K. (2010) On effectivity functions of game forms. Games and Economic Behavior 68, 512-531.

Crama, Y., Hammer, P.L. (2011) Boolean functions: theory, algorithms, and applications. Cambridge University Press, New York.

Gärdenfors, P. (1981) Rights, games and social choice. Noûs 15, 341-356. 
Harsanyi, J. (1967) Games with incomplete information played by 'Bayesian' players, parts I-III. Management Science 14, 158-182, 320-334, 486502 .

Moulin, H. (1983) The strategy of social choice. North-Holland, Amsterdam.

Moulin, H., Peleg, B. (1982) Cores of effectivity functions and implementation theory. Journal of Mathematical Economics 10, 115-145.

Peleg, B. (1998) Effectivity functions, game forms, games, and rights. Social Choice and Welfare 15, 67-80.

Peleg, B., Peters, H., Storcken, T. (2002) Nash consistent representation of constitutions: a reaction to the Gibbard paradox. Mathematical Social Sciences 43, 267-287.

Peleg, B., Peters, H. (2010) Strategic social choice: stable representations of constitutions. Springer-Verlag, Berlin Heidelberg.

Peleg, B., Zamir, S. (2013) Representation of constitutions under incomplete information. Center for the Study of Rationality. Discussion paper 634 .

\section{A Construction of the representation in Exam- ple 5.9}

The construction proceeds in several steps.

Step 1 We modify $G_{0}=\left(\Sigma^{1}, \Sigma^{2}, \Sigma^{3} ; \pi ; A\right)$ from Section 3 to a game form $G_{3}=\left(\Sigma_{3}^{1}, \Sigma_{3}^{2}, \Sigma_{3}^{3} ; \pi_{3} ; A\right)$, as follows. Let $\tilde{T}=\left(T^{1}, T^{2}\right)$. The set of actions of $i \in N$ is the set $\Sigma_{3}^{i}=\left\{(f, r, k, \phi) \mid f \in F^{i} \cup W^{\tilde{T}}, r \in T^{i}, k \in\right.$ $\{1, \ldots,|A|\}$ and $\phi \in \Phi\}$. Here, $\Phi$ is the set of all selections, as in the game form $G_{1}$ in the proof of Theorem 5.2 .

Let $\sigma=\left(\sigma^{1}, \sigma^{2}, \sigma^{3}\right) \in \Sigma_{3}^{N}$, where $\sigma^{i}=\left(f^{i}, r^{i}, k^{i}, \phi^{i}\right) \in \Sigma_{3}^{i}$ for $i \in N$. Define $K=\left\{i \in N \mid f^{i} \in F^{i}\right\}$ and $L^{R^{\tilde{T}}}=\left\{i \in N \mid f^{i}=R^{\tilde{T}}\right\}$ for all $R_{\tilde{T}}^{\tilde{T}} \in W_{\tilde{T}}^{\tilde{T}}$. Note that every $i \in N$ is either in $K$ or in $L^{R^{\tilde{T}}}$ for some $R^{\tilde{T}} \in W^{\tilde{T}}$. We define $\pi_{3}(\sigma)$ as follows.

- If $\left|L^{R^{\tilde{T}}}\right|<2$ for all $R^{\tilde{T}} \in W^{\tilde{T}}$, then $\pi_{3}(\sigma)=\pi(\sigma)$ with $\pi$ as in $G_{0}$ (see Section 3 ). 
- If $\left|L^{R^{\tilde{T}}}\right| \geq 2$ for some $R^{\tilde{T}} \in W^{\tilde{T}}$, then we distinguish two cases. Assume without loss of generality that $R^{t^{1,1}}$ orders the elements of $A$ as abcde.

(i) There are $x, y, z \in A$, with $x \neq y, x \neq z$ and $y \neq z$, such that $x R^{t^{1,1}} z$ and $y R^{t^{1,1}} z$, and $x R^{t^{1,2}} z$ and $y R^{t^{1,2}} z$. Then we define

$$
\pi_{3}(\sigma)= \begin{cases}x & \text { if }\left[L^{R^{\tilde{T}}}=\{1,2\} \text { or } L^{R^{\tilde{T}}}=N\right] \text { and } x R^{r^{2}} y . \\ y & \text { if }\left[L^{R^{\tilde{T}}}=\{1,2\} \text { or } L^{R^{\tilde{T}}}=N\right] \text { and } y P^{r^{2}} x . \\ \phi^{2}(\{x, y\}) & \text { if } L^{R^{\tilde{T}}}=\{1,3\} . \\ \phi^{1}(\{x, z\}) & \text { if } L^{R^{\tilde{T}}}=\{2,3\} \text { and } x R^{r^{2}} y . \\ \phi^{1}(\{y, z\}) & \text { if } L^{R^{\tilde{T}}}=\{2,3\} \text { and } y P^{r^{2}} z .\end{cases}
$$

(ii) There are $x, y \in\{a, b, c\}$, with $x \neq y$, such that $d R^{t^{1,2}} x$ and $d R^{t^{1,2}} y$, and $e R^{t^{1,2}} x$ and $e R^{t^{1,2}} y$. Then we define

$$
\pi_{3}(\sigma)= \begin{cases}x & \text { if } L^{R^{\tilde{T}}} \in\{\{1,2\}, N\}, r^{1}=t^{1,1}, \text { and } x R^{r^{2}} y . \\ y & \text { if } L^{R^{\tilde{T}}} \in\{\{1,2\}, N\}, r^{1}=t^{1,1}, \text { and } y P^{r^{2}} x . \\ d & \text { if } L^{R^{\tilde{T}}} \in\{\{1,2\}, N\}, r^{1}=t^{1,2}, \text { and } d R^{r^{2}} e . \\ e & \text { if } L^{R^{\tilde{T}}} \in\{\{1,2\}, N\}, r^{1}=t^{1,2}, \text { and } e P^{r^{2}} d . \\ \phi^{2}(\{x, y\}) & \text { if } L^{R^{\tilde{T}}}=\{1,3\} \text { and } r^{1}=t^{1,1} . \\ \phi^{2}(\{d, e\}) & \text { if } L^{R^{\tilde{T}}}=\{1,3\} \text { and } r^{1}=t^{1,2} . \\ \phi^{1}(\{x, d\}) & \text { if } L^{R^{\tilde{T}}}=\{2,3\}, x R^{r^{2}} y, \text { and } d R^{r^{2}} e . \\ \phi^{1}(\{x, e\}) & \text { if } L^{R^{\tilde{T}}}=\{2,3\}, x R^{r^{2}} y, \text { and } e P^{r^{2}} d . \\ \phi^{1}(\{y, d\}) & \text { if } L^{R^{\tilde{T}}}=\{2,3\}, y P^{r^{2}} x, \text { and } d R^{r^{2}} e . \\ \phi^{1}(\{y, e\}) & \text { if } L^{R^{\tilde{T}}}=\{2,3\}, y P^{r^{2}} x, \text { and } e P^{r^{2}} d .\end{cases}
$$

We now show that the game form $G_{3}$ is properly defined, in particular, that the cases (i) and (ii) cover all preference orderings. Suppose we are not in case (i). Then there are no $x, y, z \in A$, with $x \neq y, x \neq z$ and $y \neq z$, such that $x R^{t^{1,1}} z$ and $y R^{t^{1,1}} z$, and $x R^{t^{1,2}} z$ and $y R^{t^{1,2}} z$. For $z=e$, this implies that there is at most one alternative preferred to $e$ for $t^{1,2}$.

If $d R^{t^{1,2}} e$, then this means $e P^{t^{1,2}} a, e P^{t^{1,2}} b$ and $e P^{t^{1,2}} c$ and by transitivity, $d P^{t^{1,2}} a, d P^{t^{1,2}} b$ and $d P^{t^{1,2}} c$. Hence we are in case (ii).

If $e P^{t^{1,2}} d$, then for $z=d$, the supposition that we are not in case (i) implies that there is at most one alternative from $\{a, b, c\}$ preferred to $d$ for $t^{1,2}$. This means there are at least two alternatives from $\{a, b, c\}$ to which $d$ is strictly preferred. By transitivity, $e$ is also strictly preferred to these two alternatives and hence we are in case (ii). 
Step 2 We show that $G_{3}$ is a representation of $E$. First, we show that $E(S) \subseteq E^{G_{3}}(S)$ for all $S \in P_{0}(N)$. Let $S \in P_{0}(N)$ and $B \in E(S)$. We distinguish three cases:

(a) $|S| \geq 2$. Choose $\sigma^{S}$ such that $f^{i}=(S, B)$ for all $i \in S$. Then the coalition $S$ is formed and hence by definition of $\pi_{3}, \pi_{3}\left(\sigma^{S}, \tau^{N \backslash S}\right) \in B$ for all $\tau^{N \backslash S} \in \Sigma_{3}^{N \backslash S}$. So, $B\left(\sigma^{S}\right) \subseteq B$.

(b) $S=\{i\}$, with $i=1,2$. Choose $\sigma^{i}$ such that $f^{i}=(\{i\}, B)$ and $\phi^{i}$ is such that $\phi^{i}\left(B^{\prime}\right) \in B$ for all $B^{\prime}$ with $B^{\prime} \cap B \neq \emptyset$.

If $\tau^{N \backslash\{i\}}$ is such that $f^{j}=R^{\tilde{T}}$ for all $j \in N \backslash\{i\}$, then player $i$ is able to choose from $\{x, y\}$ for some $x, y \in A$ and $x \neq y$. Since $\{x, y\} \in E(N \backslash\{i\})$, superadditivity implies $A \backslash\{x, y\} \notin E(1)$. Hence $B \cap\{x, y\} \neq \emptyset$, which means that $\phi^{i}$ will select an alternative from $B$.

For all other $\tau^{N \backslash\{i\}} \in \Sigma_{3}^{N \backslash\{i\}}$, it is sufficient to note that coalition $\{i\}$ forms. Thus, by definition of $\pi_{3}, \pi_{3}\left(\sigma^{i}, \tau^{N \backslash\{i\}}\right) \in B$ for all $\tau^{N \backslash\{i\}} \in \Sigma_{3}^{N \backslash\{i\}}$.

(c) $S=\{3\}$. It is sufficient to note that since $E(\{1,2\})=P_{0}(A)$, superadditivity implies that $E(3)=\{A\}$.

In order to prove the converse inclusion, let $S \in P_{0}(N)$ and $B \in E^{G_{3}}(S)$. Since $E(N)=P_{0}(A)$ and $A \in E(S)$ for all $S \in P_{0}(N)$, we can assume that $S \neq N$ and $B \neq A$. We show that $B \in E(S)$. Let $\sigma^{S} \in \Sigma^{S}$ be such that $B\left(\sigma^{S}\right) \subseteq B$. We only treat the case $|S|=2$ and $\sigma^{S}$ is such that $f^{i}=R^{\tilde{T}}$ for some $R^{\tilde{T}} \in W^{\tilde{T}}$ and for all $i \in S$. For all other cases, it is sufficient to note that $G_{3}$ is an extension of $G_{0}$.

If $3 \notin S$, then it is sufficient to note that $E(S)=E(\{1,2\})=P_{0}(A)$.

If $3 \in S$, then by construction $B\left(\sigma^{S}\right)=\{x, y\}$ for some $x, y \in A$ and $x \neq y$. Since $\{x, y\} \in E(S)$, monotonicity implies that $B \in E(S)$.

Step 3 It remains to prove that $G_{3}$ is ex post consistent. Let $R^{T} \in W^{T}$. Consider the following strategy profile $s \in S^{N}$. For every $t^{i} \in T^{i}$ and for all $i \in N, s\left(t^{i}\right)=\left(R^{\tilde{T}}, t^{i}, 1, \phi^{i}\right)$, with $\phi^{i}$ chosen arbitrarily.

For case (i) in Step 1, all $t^{1} \in T^{1}$ prefer $x$ to $z$ and $y$ to $z$ and all $t^{2} \in T^{2}$ obtain their preferred element from $\{x, y\}$. Hence these types have no incentive to deviate.

For case (ii) in Step $1, t^{1,1} \in T^{1}$ prefers $x$ and $y$ to $d$ and $e$, whereas $t^{1,2} \in T^{1}$ prefers $d$ and $e$ to $x$ and $y$. Moreover, if $r^{1}=t^{1,1}$, all $t^{2} \in T^{2}$ obtain their preferred element from $\{x, y\}$, and if $r^{1}=t^{1,2}$, all $t^{2} \in T^{2}$ obtain their preferred element from $\{d, e\}$. Thus, none of these types is able to improve.

Since no $t^{3} \in T^{3}$ is able to affect the outcome under this strategy profile, we have an EPE. 